\title{
sciendo
}

Transport and Telecommunication, 2019, volume 20, no. 2, 114-122

Transport and Telecommunication Institute, Lomonosova 1, Riga, LV-1019, Latvia

DOI 10.2478/ttj-2019-0010

\section{REAL-TIME FREEWAY TRAFFIC STATE ESTIMATION BASED ON THE SECOND-ORDER DIVIDED DIFFERENCE KALMAN FILTER}

\author{
Asmâa Ouessai ${ }^{1}$, Mokhtar Keche ${ }^{2}$ \\ Signals and Images Laboratory, Department of Electronics, \\ University of Sciences and Technology USTO-MB, B.P. 1505, El M'Naouar - Bir el Djir- \\ Oran, Algeria \\ 1Ouessai.as@gmail.com,2m_keche@yahoo.com
}

\begin{abstract}
Reliable road traffic state identification systems should be designed to provide accurate traffic state information anywhere and anytime. In this paper we propose a road traffic classification system, based on traffic variables estimated using the second order Divided Difference Kalman Filter (DDKF2). This filter is compared with the Extended Kalman Filter (EKF) using both simulated and real-world dataset of highway traffic. Monte-Carlo simulations indicate that the DDKF2 outperforms the EKF filter in terms of parameters estimation error. The real-word evaluation of the DDKF2 filter in terms of classification rate confirms that this filter is promising for real-world traffic state identification systems.
\end{abstract}

Keywords: Divided Difference Kalman filter, Extended Kalman filter, road traffic estimation, road traffic classification, support vector machine

\section{Introduction}

Real-time road traffic information is very requested nowadays by road users, because it helps them to choose the best road route. Efficient Intelligent Transport Systems (ITS) and reliable information are essentials for this task. However, lack of or wrong information about the traffic state can put the drivers' safety at a serious risk, since it is known that the effects of congestion on safety depend on the extent to which drivers are surprised by the congestion (Marchesini and Weijermars, 2010). This is especially true if the automatic road traffic monitoring system estimates that the road is free, while it is congested in fact, which can lead to rear-end crash (Marchesini and Weijermars, 2010), due to the difference between the speed of the incoming vehicle and the estimated mean traffic speed.

One solution proposed to overcome these problems is to rely on systems based on efficient traffic estimation methods. Traffic estimation refers to the estimation of the traffic parameters (speed, density and flows), based on a limited number of traffic variables measured by one or more detectors.

Since the early 1970s, considerable efforts have been spent to design systems for estimating road traffic state (Knapp, 1972; Nahi and Trivedi, 1973; Grewal and Payne, 1976). Most of the recent works dealing with this problem have proposed estimation algorithms based on the nonlinear extensions of the Kalman filter (Seo et al., 2017).

The extended Kalman filter (EKF) has been widely used in traffic estimation systems (Wang and Papageorgiou, 2003, 2005; Wang et al., 2008; Yuan et al., 2014) and over large road traffic networks. However, its principal drawback is its divergence that may be caused by the linearization procedure. Pueboobpaphan and Nakatsuji (2006) used the unscented Kalman filter (UKF) as an alternative to the EKF filter, for road traffic estimation. The UKF performance was compared to that of the particle filter proposed by Mihaylova and Boel (2004); the authors concluded that the UKF is a promising method for traffic flow estimation, requiring a low computational cost (Mihaylova et al., 2006). On the other hand, a comparison of several configurations of the UKF filter and its classic competitor, the EKF filter, for freeway traffic state estimation, was presented by Hegyi et al. (2006), this comparison is based on the performance regarding state estimation, parameter estimation, joint estimation and dual estimation. The main conclusions are that the performances of the extended Kalman filter and the unscented Kalman filter are nearly similar. The ensemble Kalman filter (EnKF) was proposed by Work et al. (2008) and Seo et al. (2015) for estimating the velocity field on a highway; this filter uses Monte Carlo simulations to 
overcome the EKF shortcomings. Thai and Bayen (2015) presented a complexity analysis of both the $\mathrm{EKF}$ and the EnKF filters for traffic density estimation. The EKF was found to be significantly faster than the EnKF with 100 samples.

In this paper, we propose to use the second-order divided difference Kalman (DDKF2) filter for road traffic parameters estimation. The DDKF2 filter was proposed by Norgaard et al. (2000); it is based on the second order polynomial approximation, derived with Stirling's interpolation formula, in contrast to the EKF, which is based on polynomial approximation obtained with Taylor formula, it requires no derivatives calculation.

The DDKF and the DDKF2 filters performances have been investigated in many applications (Jwo et al., 2010; Ahmadi et al., 2012); the results of investigations indicate that these filters outperform the EKF filter and the UKF filter in most of cases. The first and the second order divided difference filters were compared in (Ahmadi et al., 2012), based on the estimation accuracy; the main conclusions have recommended the DDKF2 filter for many applications (Salhi and Bouani, 2017; Wu and Han, 2008). This filter is adopted in this paper and compared to the EKF filter, in terms of traffic parameters estimation accuracy.

The rest of this paper is organized as follows: Firstly, the proposed approach is detailed in section 2. In section 3, the dynamic traffic model is described, and in section 4, the main steps of the EKF and the DDKF2 filters are presented. Section 5 is devoted to the presentation and discussion of the experimental results, and finally, some conclusions are drawn in section 6.

\section{The Proposed Approach}

The proposed framework is presented in the Figure 1. A fixed camera sensor is used in this work to record the traffic videos that are properly processed to automatically measure the mean traffic density and the mean traffic speed using image-processing techniques; then a macroscopic traffic flow model is used to describe the dynamic behaviour of the traffic flow along a freeway stretch. The DDKF2 filter is employed to estimate the mean traffic speed and the mean traffic density. Based on these estimates, the SVM classifies the traffic state into two main classes: free and congested.

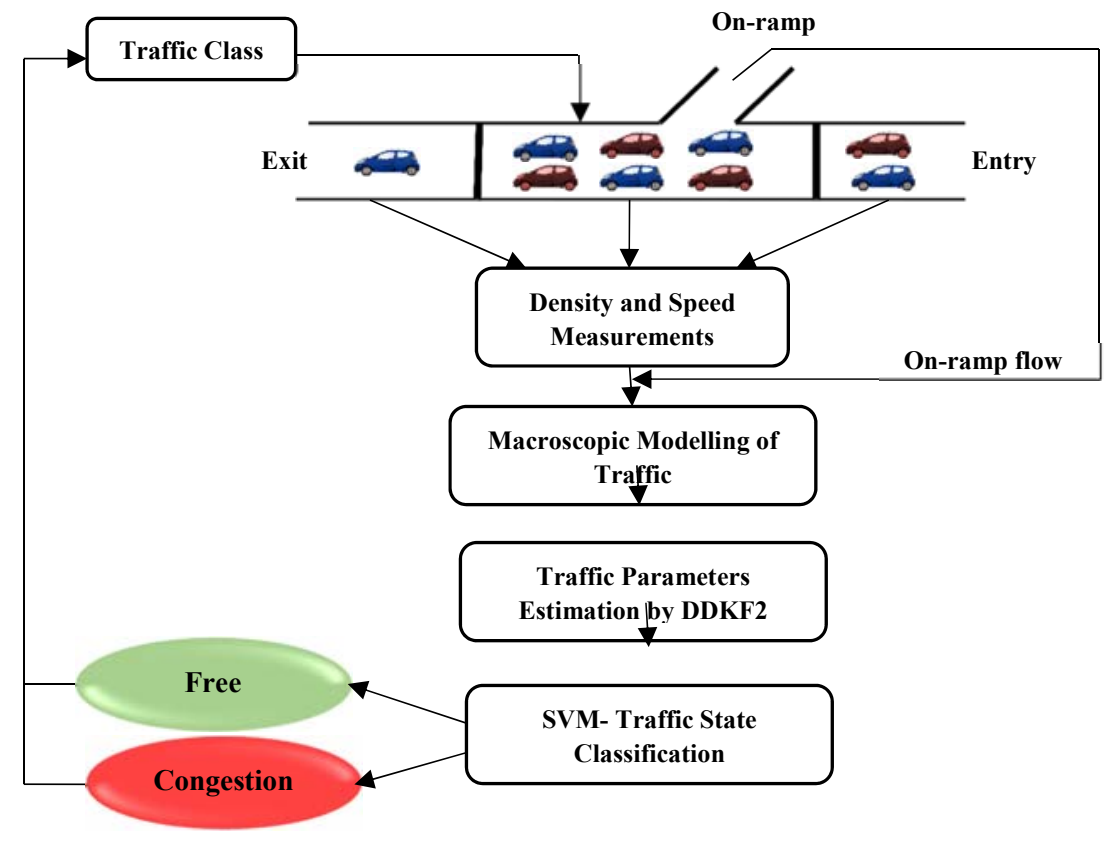

Figure 1. The proposed approach

In the following sections, we will describe the macroscopic model and the traffic estimation methods. The traffic measurements and the classification framework will be briefly described in the results section. 


\section{Macroscopic Freeway Traffic Flow Model}

To describe the dynamic behaviour of traffic flow in a given freeway section of the road, we employ the second order macroscopic traffic flow model described by Wang and Papageorgiou (2003, 2005).

Based on this model, the freeway is divided into a number of sections; the length of section $i$ is denoted $\Delta_{i}$. The time discretization is based on a model time step, denoted $\mathrm{T}$. The aggregated traffic parameters are calculated according to the following equations:

$$
\begin{aligned}
& \rho_{i}(k+1)=\rho_{i}(k)+\frac{T}{\Delta_{i} \lambda_{i}}\left[q_{i-1}(k)-q_{i}(k)+r_{i}(k)\right], \\
& \begin{array}{l}
v_{i}(k+1)=v_{i}(k)+\frac{T}{\tau}\left[V\left(\rho_{i}(k)\right)-v_{i}(k)\right]+\frac{T}{\Delta_{i}} v_{i}(k)\left[v_{i-1}(k)-v_{i}(k)\right]-\frac{v T}{\tau \Delta_{i}} \frac{\left[\rho_{i+1}(k)-\rho_{i}(k)\right]}{\rho_{i}(k)+x} \\
\quad-\frac{\delta T}{\Delta_{i} \lambda_{i}} \frac{r_{i}(k) v_{i}(k)}{\rho_{i}(k)+x}+\xi_{i}^{v}(k)
\end{array} \\
& \begin{aligned}
V(\rho)=v_{f} \exp \left[-\frac{1}{a}\left(\frac{\rho}{\rho_{c}}\right)^{a}\right], \\
q_{i}(k)=\rho_{i}(k) \cdot v_{i}(k) \cdot \lambda_{i}+\xi_{i}^{q}(k),
\end{aligned}
\end{aligned}
$$

where:

$\rho_{i}(k)$ - is the traffic density in section $i$ at time $\mathrm{kT}$,

$v_{i}(k)$ - is the average speed in section $i$ at time $\mathrm{kT}$,

$q_{i}(k)$ - is the traffic flow in section $i$ at time instant $\mathrm{kT}$,

$r_{i}(k)$ - is the on-ramp inflow,

$v_{f}, \rho_{c r}$ - are, respectively, the free speed and the critical density,

$a$ - is an exponent parameter and $\lambda_{i}$ denotes the number of lanes in section $i$,

$\tau, \nu$ and $\delta$ - are, respectively, a time constant, an anticipation constant, and an on-ramp constant, $x$ - is a constant parameter used to keep the third and fourth terms limited when $\rho_{i}$ becomes small,

$\xi_{i}^{v}(k)$ and $\xi_{i}^{q}(k)$ - are zero mean white Gaussian noise, added to reflect the modelling inaccuracies.

Eq. (1) describes the conservation of vehicles; it is thus considered as not corrupted by noise.

\section{The Extended Kalman Filter and the Divided Difference Kalman Filter for Traffic Parameters Estimation}

In order to evaluate the DDKF2 filter for road traffic estimation, we compare its estimation accuracy to that of the EKF; both filters are described in this section.

\subsection{The EKF filter}

The EKF filter (Bar-Shalom et al., 2001) is well documented; we thus just summarize the algorithm steps in the following figure:

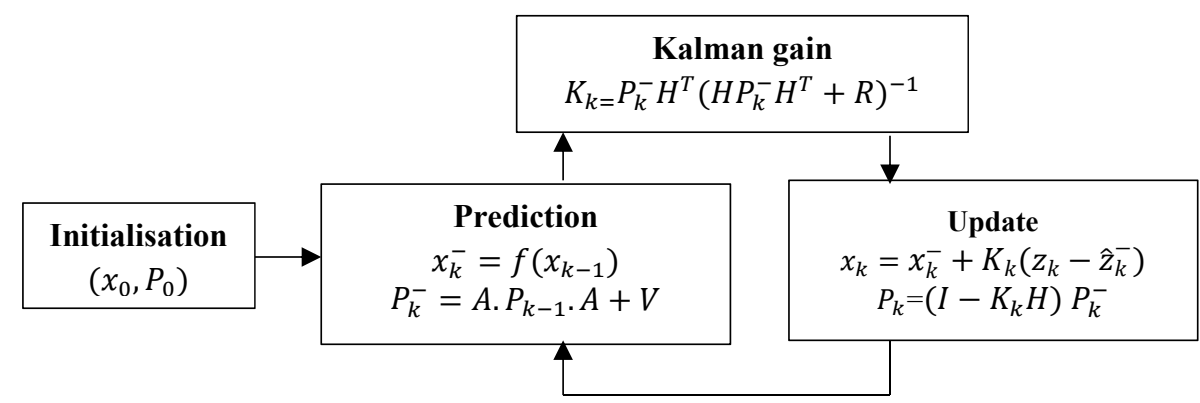

Figure 2. EKF filter

In this figure $\left(x_{0}, P_{0}\right)$ is, respectively, the initial state space vector and the corresponding covariance matrix, with $x_{0}=\left[\begin{array}{l}\rho_{0} \\ v_{0}\end{array}\right]$; 
- $f$ is the nonlinear function that relates the state vectors $x(k)$ and $x(k-1)$, with $\left[\begin{array}{l}\rho_{i}(k+1) \\ v_{i}(k+1)\end{array}\right]=f\left[\begin{array}{l}\rho_{i}(k) \\ v_{i}(k)\end{array}\right]+v(k), v(k)$ being the process noise.

- $\boldsymbol{A}$ denotes the matrix of the partial derivatives of $f$ with respect to $x$, defined as: $\boldsymbol{A}=\frac{\partial f}{\partial x}$,

- $H$ denotes the measurement matrix, given by: $\boldsymbol{H}=\left[\begin{array}{ll}1 & 0 \\ 0 & 1\end{array}\right]$,

- $\boldsymbol{R}$ and $\boldsymbol{V}$ represent the measurement noise and the process noise covariance matrices.

\subsection{The DDKF2 filter}

The DDKF2 filter is based on the multi variables extension of Stirling's interpolation formula, rather than the derivative based Taylor series approximation. The DDKF2 filter is simple to implement, as no derivatives calculation is required. The DDKF2 algorithm designed for road traffic estimation consists of the following 5 steps:

- Initialization:

Set $x_{0}, P_{0}, S^{x}$ where $S^{x}$ is the Cholesky factorization of the $P_{0}$ matrix: $S^{x}=\operatorname{chol}\left(P_{0}\right)$;

- Calculation of the predicted state vector, $x_{k}$, and its covariance matrix $P_{k}$ :

$x_{k}=\frac{h^{2}-n_{x}}{h^{2}} f\left(x_{k-1}\right)+\frac{1}{2 h^{2}} \sum_{i=1}^{n_{x}}\left[f\left(x_{k-1}+s_{k-1}^{x, j}\right)+f\left(x_{k-1}-s_{k-1}^{x, j}\right)\right]$,

where $x_{k}=\left[\begin{array}{l}v(k) \\ \rho(k)\end{array}\right]$, with $v(k)$ and $\rho(k)$ being, respectively, the mean traffic speed and the mean traffic density at time $\mathrm{k} ; f$ is given in Eq.(1) and Eq.(2); $n_{x}$ is the state vector dimension; $h$ is a constant whose choice is discussed in (Norgaard et al., 2000) and $s_{k-1}^{x, j}$ is the jth column vector of matrix $S^{x}$;

$$
\begin{gathered}
P_{k}=\frac{1}{4 h^{2}} \sum_{i=1}^{n_{x}}\left(\left[f\left(x_{k-1}+s_{k-1}^{x, j}\right)+f\left(x_{k-1}-s_{k-1}^{x, j}\right)\right] \times\left[f\left(x_{k-1}+s_{k-1}^{x, j}\right)+f\left(x_{k-1}-s_{k-1}^{x, j}\right)\right]^{\prime}\right) \\
+\frac{h^{2}-1}{4 h^{4}} \sum_{i=1}^{n_{x}}\left(\left[f\left(x_{k-1}+s_{k-1}^{x, j}\right)+f\left(x_{k-1}-s_{k-1}^{x, j}\right)-2 f\left(x_{k-1}\right)\right] \times\right. \\
\left.\left[f\left(x_{k-1}+s_{k-1}^{x, j}\right)+f\left(x_{k-1}-s_{k-1}^{x, j}\right)-2 f\left(x_{k-1}\right)\right]^{\prime}\right) ;
\end{gathered}
$$

- Measurement prediction

$\hat{z}_{x}=H x_{k}$,

where $\boldsymbol{H}$ is the measurement matrix defined previously;

- Kalman gain calculation

$$
K_{k}=P_{k} H^{T}\left(H P_{k} H^{T}+R\right)^{-1} ;
$$

- Update

$$
\begin{aligned}
& x_{k+1}=x_{k}+K_{k}\left(z_{k}-\hat{z}_{x}\right), \\
& P_{k+1}=\left(I-K_{k} H\right) P_{k}, \\
& S^{x}=\operatorname{chol}\left(P_{k}\right),
\end{aligned}
$$

where $z_{k}$ is the measurment vector, whose elements are the measured mean traffic speed and the measured mean traffic density, at time instant k, and $\boldsymbol{I}$ is the identity matrix.

\section{Results and Discussion}

\subsection{Evaluation using simulated data}

Firstly, we implement and evaluate the proposed traffic state estimation system using simulated data, generated by means of the macroscopic model presented in section 3 .

The unknown traffic model parameters presented in Eq. (3), namely the free speed $v_{f}$ and the density at capacity $\rho_{c}$, were calibrated by minimizing the dispersion between the model outputs and the 
sensor measurements, using the Complex based optimization algorithm, developed by Box (1956). This algorithm is widely used in the literature, especially for road traffic modelling (Kotsialos and Papageorgiou, 2002; Ouessai and Keche, 2015). The modelling task was achieved by using the measured parameters of the exit section obtained from the real time video traffic data set, presented in section 5.2.1. The obtained functional form is given below:

$v=\left(2.76 * 10^{4}\right) * \exp \left(-0.5 *(\rho / 3)^{2}\right)$.

The macroscopic model is used to generate the simulated real time traffic speed and density in the middle section presented in Figure 1. Then, the simulated measurements were obtained by adding random noises, with means 0.5 and 1000 , to the real speed and density, respectively.

The selected parameters for the macroscopic dynamic model are: $\lambda_{i}=1, \Delta_{i}=320$ pixels, $\mathrm{T}=0.01$ hour, $\tau=0.25$ hour, $v=35$ pixel $^{2} /$ hour, $\mathrm{x}=13 . \delta=1.4$.

We carried out 100 Monte-Carlo runs to estimate the mean traffic speed and density in the middle section using the EKF and the DDKF2 filters.

The measurement and the process noise covariance matrices for the both filters were, respectively, set to: $\boldsymbol{R}=\left[\begin{array}{cc}0.25 & 0.1 \\ 0.1 & 1 e 6\end{array}\right]$ and $\boldsymbol{V}=\left[\begin{array}{cc}0.01 & 0.01 \\ 0.01 & 1\end{array}\right]$. For the DDKF2 filter we take $h=10$.

All the experiments, in this paper, are conducted in MATLAB environment (version R2017b), running on a laptop with $2.20 \mathrm{GH}_{\mathrm{Z}}$ Intel ${ }^{\circledR}$ Core i7-2670QM CPU and a $6.00 \mathrm{~GB}$ of RAM.

The obtained results of traffic parameters estimation using EKF and DDKFS filters are presented in the Figures 3 and 4.
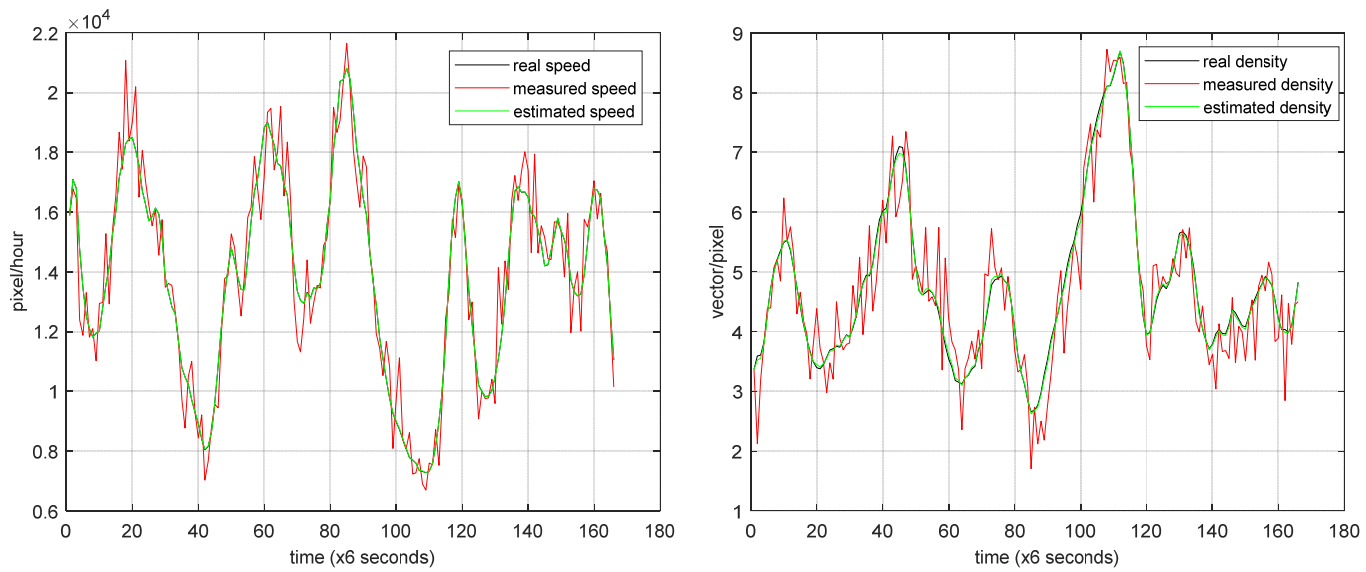

Figure 3. Traffic parameters estimation using the EKF filter
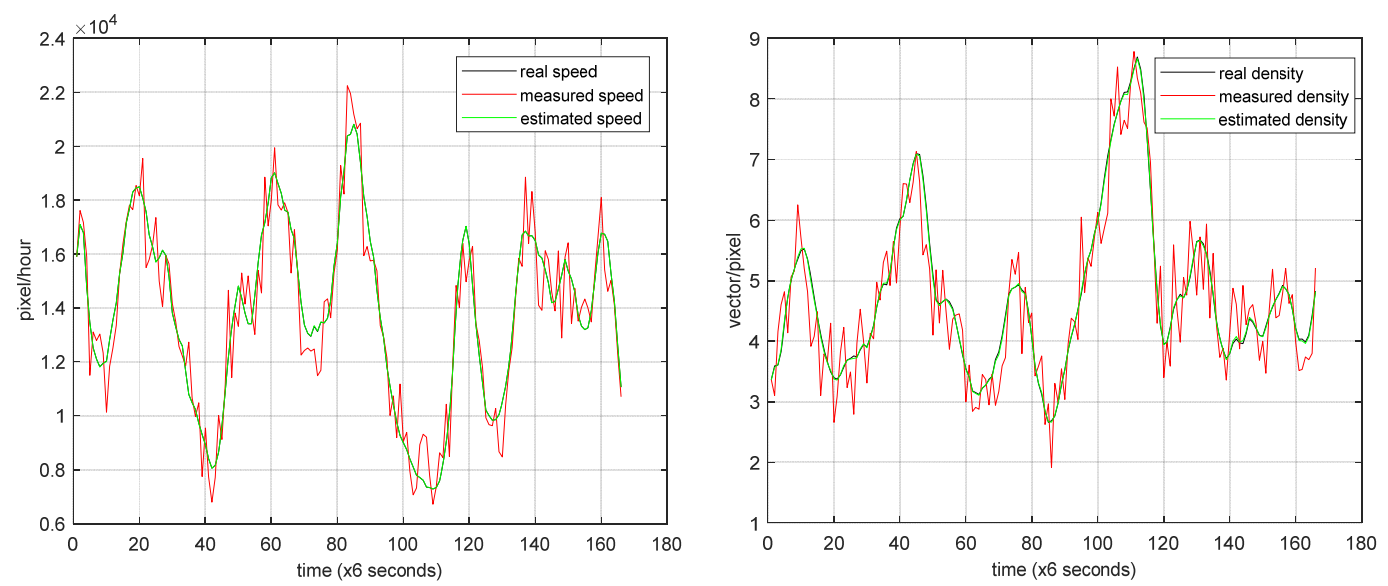

Figure 4. Traffic parameters estimation using the DDKF2 filter 
Figure 5 shows a comparison between the EKF and the DDKF2, based on the Root Mean Square Error (RMSE) of the density and speed.
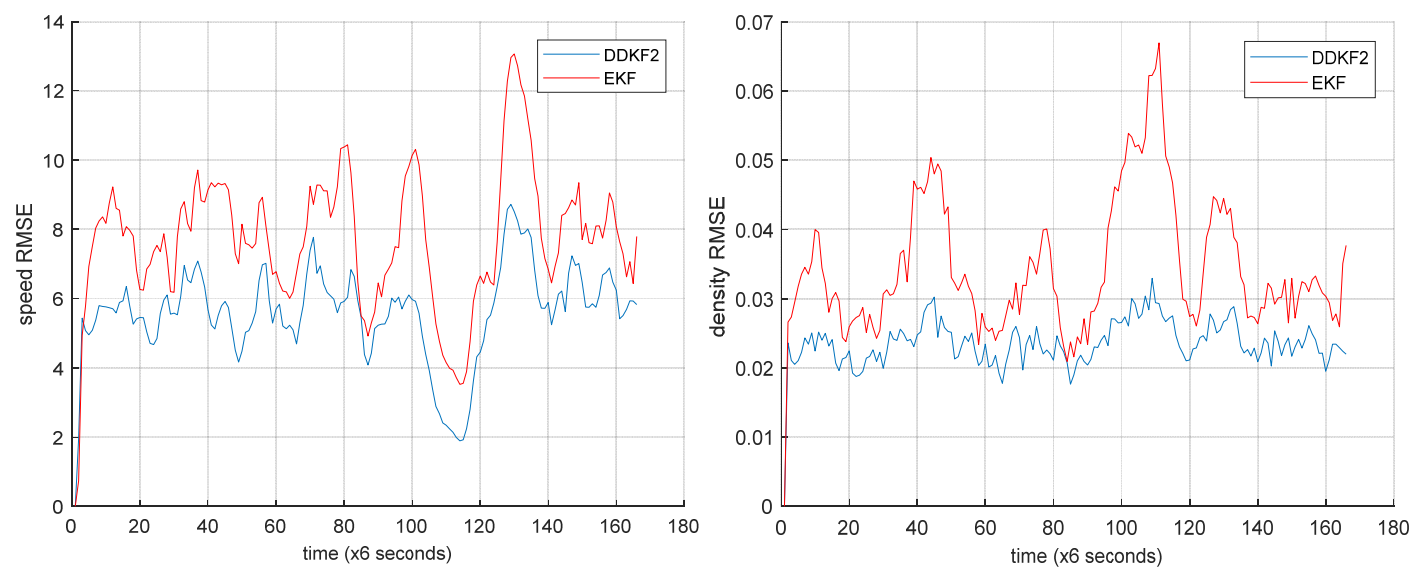

Figure 5. DDKF2 and EKF estimation errors

The Table 1 shows the Mean Absolute Percentage Error (MAPE), obtained by the two filters. The MAPE is defined by:

MAPE $=\frac{1}{N} \sum_{k=1}^{N}\left[\frac{\left|x_{\text {est }}(k)-x_{\text {real }}(k)\right|}{x_{\text {real }}(k)}\right] * 100$,

where $x_{\text {est }}(k)$ and $x_{\text {real }}(k)$ are, respectively, the variables (speed or density) estimated by the EKF or the DDKF2 and the their real values.

Table 1. Estimation errors

\begin{tabular}{|c|c|c|}
\cline { 2 - 3 } \multicolumn{1}{c|}{} & \multicolumn{2}{|c|}{ MAPE } \\
\cline { 2 - 3 } & Speed & Density \\
\hline EKF & 0.0038 & $3.0765 \mathrm{e}-04$ \\
\hline DDKF2 & 0.0024 & $1.7485 \mathrm{e}-04$ \\
\hline
\end{tabular}

These results show that the DDKF2 filter is able to estimate the traffic speed and density more accurately than the EKF filter.

As traffic speed and density are the essential traffic congestion indicators (Al-Sobky and Ragab, 2016), the DDKF2 is expected to be better than the EKF for traffic state classification.

\subsection{Evaluation of the traffic state estimators using a real time freeway traffic dataset}

In the following, we present the results of the evaluation of the EKF and DDKF2 traffic state estimators by means of a real time freeway traffic dataset, described below.

\subsubsection{Video dataset}

The existing public UCSD (2005) dataset is unsuitable for our application, since it contains videos from only one camera in one section of the road. The most suitable dataset that we could find for our problem is the US Highway 101 (US 101) video dataset, also known as the Hollywood Freeway, which was collected by researchers of the NGSIM program in Los Angeles (NGSIM, 2005). This dataset contains videos from several cameras, which cover a large-scale highway; each camera is mounted on one section of the highway. The extent of the US 101 studied area is shown in Figure 6. The traffic parameters inside section 2, estimated using the EKF or the DDKF2 filters, are fed to a SVM, to classify the traffic state as free or congested. 


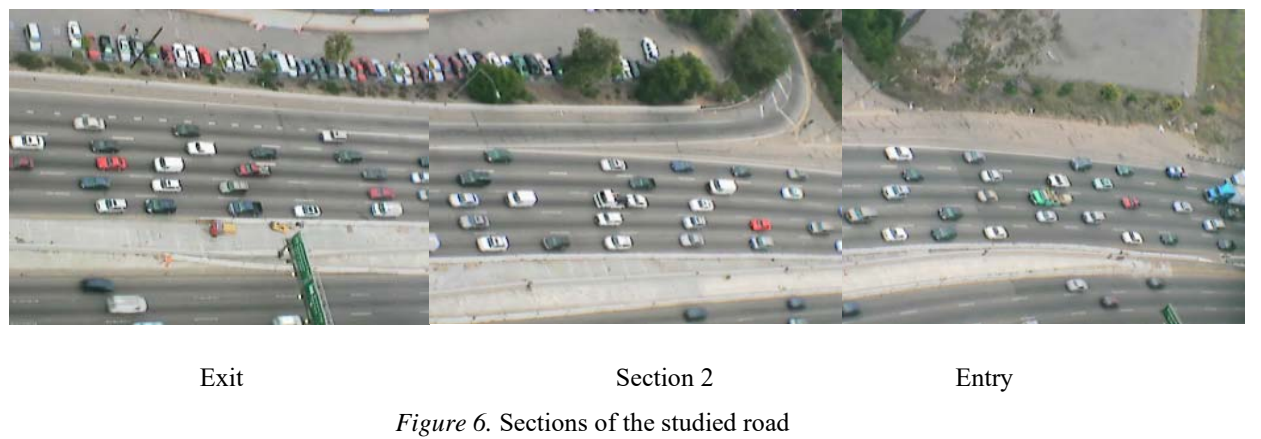

Firstly, each video footage of $15 \mathrm{~min}$ is divided into sequences of 5 seconds; in total, we have 166 video sequences for each section of the road, each video having a resolution of $320 \times 240$ pixels, with 58 to 61 frames captured at 10 frames per second. Secondly, the hand-labelled ground truth is established to describe the traffic state in section 2; the 166 videos are grouped into two classes: 109 videos of heavy (congested) traffic (slow or stop and go speeds), and 57 videos of light (free) traffic (normal speed). In Figure 7, we show a representative set of clips from this database.

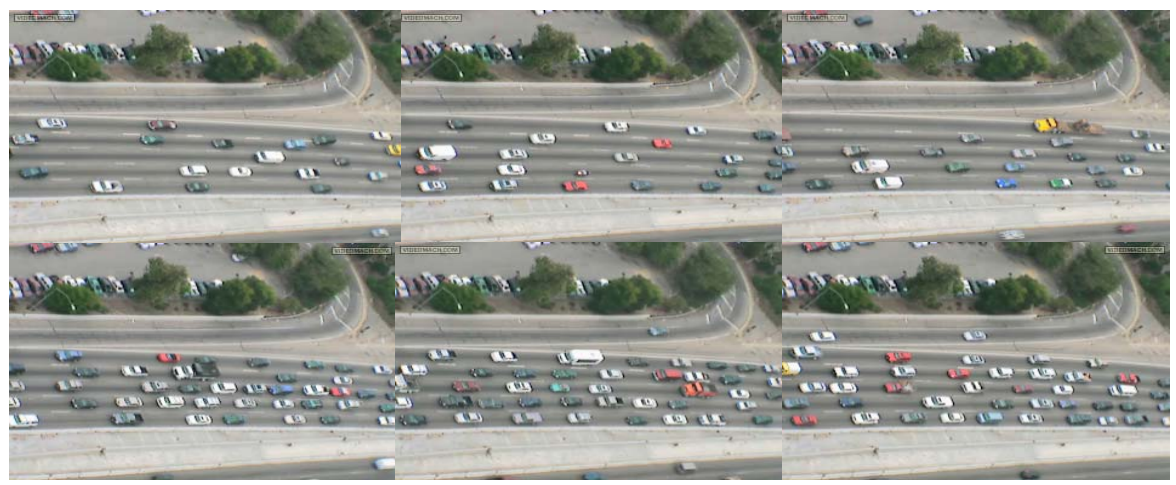

Figure 7. Example of frames from the US 101 traffic video dataset. The sample frames depict two traffic conditions in section 2 , coarsely categorized as light (top row) and congested (bottom row)

\subsubsection{Traffic data collection from video sequences}

The collection of the raw variables of the road traffic (mean traffic speed and mean traffic flow) from the video sequences in each road section was performed automatically, using the image processing method presented in (Ouessai et al., 2013a; 2013b). This method was evaluated by Ouessai et al. (2013b), using the UCSD dataset, in order to accurately extract the macroscopic traffic variables, without the need for motion detection and tracking.

The mean traffic density was obtained using the fundamental relation: $\rho=q / v$.

The side road in section 2 was separately segmented and its traffic flow was measured using the same method.

\subsubsection{Implementation of the EKF and the DDKF2 filters}

The parameters used in the EKF and DDKF2 filters were tuned so that each filter achieves its best performance. The parameters of the macroscopic traffic flow model were set as follows:

$\lambda_{i}=1, \Delta_{i}=320$ pixels, $\mathrm{T}=0.01$ hour, $\tau=0.25$ hour, $v=35$ pixel $^{2} /$ hour, $\mathrm{x}=9$ for the $\mathrm{EKF}$ and $\mathrm{x}=13$ for the DDKF2, and $\delta=1.4$.

In both filters, the initial state vector was set equal to the first measurement vector, and the covariance matrix was set to: $p_{0}=\left[\begin{array}{cc}0.01 & 0.1 \\ 0.1 & 1\end{array}\right]$. 
The measurement and the process noise covariance matrices for the EKF filter were, respectively, set to: $\boldsymbol{R}=\left[\begin{array}{cc}0.015 & 1 \\ 1 & 1 e 6\end{array}\right]$, and $\boldsymbol{V}=\left[\begin{array}{cc}0.1 & 0.1 \\ 0.1 & 1\end{array}\right]$, while for the DDKF2 filter they were, respectively, set to:

$\boldsymbol{R}=\left[\begin{array}{cc}0.015 & 1 \\ 1 & 7 e 3\end{array}\right]$, and $\boldsymbol{V}=\left[\begin{array}{cc}0.01 & 0.1 \\ 0.1 & 1\end{array}\right]$. For this later filter we took $h=5$

\subsubsection{SVM classification}

The SVM classifier is used in this work to classify the real-time traffic state into two classes: free and congested, these two main classes are the most needed by road users.

Using trial and error, it was found that for the SVM, a radial basis function Kernel (RBF) with $\sigma=$ 0.2 gives the best results. The entire dataset was split unequally, with $75 \%$ used for training, and $25 \%$ reserved for testing. The traffic classification results obtained with the EKF and the DDKF2 are presented in Table 2.

Table 2. Road traffic classification results

\begin{tabular}{|c|c|c|c|}
\cline { 2 - 4 } & Measurements & EKF estimates & DDKF2 estimates \\
\hline SVM Classification & $84.61 \%$ & $89.77 \%$ & $93.98 \%$ \\
\hline
\end{tabular}

These results show that the SVM classification rate, obtained with the mean traffic speed and density estimated by the DDKF2 filter, is higher than the classification rate obtained using the mean traffic speed and density estimated by the EKF filter. Both filters improve the results of classification obtained by using the raw measured parameters.

\section{Conclusion}

This paper investigated the performance of the DDKF2 filter for road traffic parameters estimation over a large-scale highway. It was compared, with the performance of the EKF filter, using both simulated and real-time data. The carried out Monte-Carlo simulations show that the DDKF2 filter has a better estimation accuracy, compared to the EKF. The real-time evaluation was performed using the US 101 video dataset. The estimated parameters were fed to a SVM classifier in order to classify the traffic state as congested or free. The classification rate obtained using the DDKF2 estimates is higher than the one obtained using the EKF estimates. Therefore, it can be stated that the DDKF2 is a promising filter for road traffic state estimation.

\section{References}

1. Ahmadi, M., Khayatian, A., Karimaghaee, P. (2012) Attitude estimation by divided difference filter in quaternion space, Acta Astronautica, 75, 95-107.

2. Al-Sobky, A.A., Ragab, M. (2016) Traffic density determination and its applications using smartphone, Alexandria Engineering Journal, 513-523.

3. Bar-Shalom, Y., Li, X.R., Kirubarajan, T. (2001) Estimation with Applications to Tracking and Navigation. John Wiley \& Sons, Inc.

4. Box, J. (1965) A new method of constrained optimization and a comparison with other methods, Computer Journal, 8, 42-52.

5. Grewal, M.S., Payne, H.J. (1976) Identification of parameters in a freeway traffic model, IEEE Transportation Systems, Man and Cybernetics, 6(3), 176-185.

6. Hegyi, A., Girimonte, D., Babuska, R., De Schutter, B. (2006) A comparison of filter configurations for freeway traffic state estimation. In: Proceedings IEEE ITSC, Toronto, ON, Canada, 1029-1034.

7. Jwo, D.J., Hsieh, M.Y., Lai, S.Y. (2010) GPS navigation processing using the quaternion-based divided difference filter", GPS Solutions, 14, 217-228.

8. Knapp, C.H. (1972) Traffic estimation and control of bottlenecks. In: Proceedings of IEEE Systems, Men and Cybernetics Conference.

9. Kotsialos, A., Papageorgiou, M. (2002) Traffic Flow Modeling of Large-Scale Motorway Networks Using the Macroscopic Modeling Tool METANET, IEEE Transactions on Intelligent Transportation Systems, 3(4), 282-292. 
10. Marchesini, P., Weijermars, W. (2010) The relationship between road safety and congestion on motorways. SWOV Institute for Road Safety Research, the Netherlands, Leidschendam.

11. Mihaylova, L., Boel, R. (2004) A particle filter for freeway traffic estimation. In: Proceedings of the 43rd IEEE Conference on Decision and Control, 2106-2111.

12. Mihaylova, L., Boel, R., Hegyi, A. (2006) An Unscented Kalman filter for freeway traffic estimation. In: Proceedings of 11th IFAC Symposium on Control in Transportation Systems, 31-36.

13. Nahi, N.E., Trivedi, A.N. (1973) Recursive estimation of traffic variables: section density and average speed, Transportation Science, 7, 269-286.

14. NGSIM dataset, (2005) - http://ops.fhwa.dot.gov/trafficanalysistools/ngsim.htm

15. Nørgaard, M., Poulsen, N. K., Ravn, O. (2000) New developments in state estimation for nonlinear systems, Automatica, 36, 1627-1638.

16. Ouessai, A., Keche, M. (2015) Development of road traffic flow model for nighttime conditions. In: Proceedings of IEEE 3rd International Conference on Control, Engineering \& Information Technology (CEIT), 1-6.

17. Ouessai, A., Keche, M., Ouamri, A. (2013a) Road traffic congestion estimation with macroscopic parameters. In: Proceedings of IEEE 11th International Symposium on Programming and systems, 29-34.

18. Ouessai, A., Keche, M., Ouamri, A. (2013b) Road traffic density estimation using microscopic and macroscopic parameters, Journal of Image and Vision Computing, 31, 887-894.

19. Pueboobpaphan, R., Nakatsuji, T. (2006) Real-time traffic state estimation on urban road network: The application of unscented Kalman filter. In: Proceedings of 9th International Conference of Application of Advanced Technology in Transportation Chicago, 542-547.

20. Salhi, H., Bouani, F. (2017) Experimental implementation of MIMO model predictive controllerbased second order divided difference filter for nonlinear systems, International. Journal of Modelling, Identification and Control, 27 (3), 191-199.

21. Seo, T., Bayen, A. M., Kusakabe, T., Asakura, Y. (2017) Traffic state estimation on highway: A comprehensive survey, Annual reviews in control, 43(1), 128-151.

22. Seo, T., Kusakabe. T., Asakura, Y. (2015) Traffic state estimation with the advanced probe vehicles using data assimilation. In: Proceedings of IEEE $18^{\text {th }}$ International Conference on Intelligent Transportation Systems, 824-830.

23. Thai, J., Bayen, A. M. (2015) State Estimation for Polyhedral Hybrid Systems and Applications to the Godunov Scheme for Highway Traffic Estimation, IEEE Transactions on Automatic Control, 60, 311-326.

24. UCSD (2005) - http://www.svcl.ucsd.edu.

25. Wang, Y., Papageorgiou, M. (2003) Motorway traffic state estimation based on extended Kalman filter. In: proceedings of European Control Conference (ECC), 1934-1939.

26. Wang, Y., Papageorgiou, M. (2005) Real-time freeway traffic state estimation based on extended Kalman filter: a general approach, Transportation Research Part B, 39(2), 141-167.

27. Wang, Y., Papageorgiou, M., Messmer, A. (2008) Real-time freeway traffic state estimation based on extended Kalman filter: Adaptive capabilities and real data testing, Transportation Research Part A, 42, 1340-1358.

28. Work, D.B., Tossavainen, O.P., Blandin, S., Tracton, K. (2008) An Ensemble Kalman Filtering approach to highway traffic estimation using GPS enabled mobile devices. In: Proceedings of the 47th IEEE Conference on Decision and Control, 5062-5068.

29. Wu, C., Han, C. (2008) Second-order Divided Difference Filter with Application to Ballistic Target Tracking. In: Proceedings of the $7^{\text {th }}$ World Congress on Intelligent Control and Automation, 63426347.

30. Yuan, Y., Van Lint, H., Van Wageningen-Kessels, F., Hoogendoorn, S. (2014) Network-wide traffic state estimation using loop detector and floating car data, Journal of Intelligent Transportation Systems, 18 (1), 41-50. 